\title{
Growth Pattern and Prevalence of Underweight and Stunting Among Rural Adolescents
}

\author{
Bisai ${ }^{1}$, Bose $K^{2}$, Ghosh $\mathrm{D}^{3}$, De $\mathrm{K}^{4}$ \\ ${ }^{1}$ Dr. Samiran Bisai, M Sc. Ph D. Post Doctoral Fellow, Department of Anthropology, ${ }^{2}$ Dr. Kaushik Bose, Associate \\ Professor, Department of Anthropology, ${ }^{3}$ Dr. Debidas Ghosh, Professor, Department of Bio - Medical Laboratory Science \\ and Management, ${ }^{4} \mathrm{Dr}$. Kankana De, Research Fellow; Department of Anthropology. All from Vidyasagar University, \\ West Bengal, Midnapore: 721102, India.
}

Address for Correspondence: Dr. Kaushik Bose, E-mail: banda@vsnl.net and kaushikbose@cantab.net

\begin{abstract}
Introduction: A cross-sectional study of 1094 (boys = 665; girls = 429) rural school children aged 1118 years of Midnapore Sadar North subdivision, Paschim Medinipur District, West Bengal, India, was undertaken to evaluate their growth pattern and nutritional status. Methodology: Anthropometric measurements including weight and height were measured following standard techniques. Underweight and stunting were used as indicators of nutritional status. Underweight and stunting were defined as weight-for-age (WAZ) and height-for-age (HAZ) <-2 z-scores, respectively, of the National Centre for Health Statistics (NCHS) guidelines. Results: Results revealed that boys were significantly heavier than girls from age 16 onwards; they were also significantly taller from age 14 years. The mean WAZ for boys and girls were -1.488 and -1.417 , respectively. The corresponding mean values for HAZ were -1.317 and -1.486 . The overall rates of underweight and stunting were $28.3 \%$ and $27.8 \%$, respectively. The prevalence of underweight was significantly higher among boys $(31.0 \%)$ than girls $(24.2 \%)$. These rates for stunting were $27.4 \%$ and $28.4 \%$, for the boys and girls. The rate of underweight and stunting was more in late adolescents (15-18 years) than early adolescents (11-14 years). In boys, the prevalence of stunting was significantly (1.5 times) more in late adolescents than early adolescents. According to the WHO classification for assessing severity of malnutrition, the rates of stunting were medium in both sexes. The rates of underweight were high and very high for girls and boys, respectively. Conclusion: In conclusion, present study provided evidence that the nutritional status of these adolescents were not satisfactory especially among late adolescents.
\end{abstract}

Key Words: Adolescent, Rural, Nutritional Status, Underweight, Stunting

\section{Introduction}

$\Delta$ dole dolescence is a significant transitional phase between childhood and adulthood of human growth and maturation after infancy. About $21 \%$ of the population of India, consists of adolescents (10-19 years), comprising one fifth of the total population of the country ${ }^{1}$. During this period, individuals gain about fifty percent of adult body weight and height growth with a unique pattern of sexual dimorphism. The nutritional status during this period is an important determinant of health outcomes. It was well documented that short stature in adolescents indicating prolonged undernutrition is associated with lower lean body mass, deficiencies in muscular strength and productivity ${ }^{2}$. In adolescent girls, short stature that carries on into adulthood is associated with many concurrent and future adverse health and pregnancy outcomes ${ }^{3,4,5}$. During childhood health and nutritional status is an important indicator of the national public health importance in the development of its future manpower ${ }^{6}$. Therefore formulation of exclusive health care of this section will cater to the health needs of onefifth population of the country?

Two most commonly used internationally recommended indicators used to evaluate nutritional status of adolescents are underweight (low weight-for- 
age) and stunting (low height-for-age). While underweight reveals low body mass relative to chronological age, which is influenced by both, a child's height and weight; stunting reflects a failure to reach linear growth potential due to sub optimal health and/or nutritional conditions ${ }^{2,8}$.

Many recent studies from India 9 ,10,11,12 including West Bengal ${ }^{13,14,15,16}$ have used anthropometry to evaluate nutritional status of adolescents. However, there is a dearth of data on the prevalence of underweight and stunting among adolescents from rural West Bengal. Therefore, we conducted a study to evaluate growth pattern and levels of underweight and stunting, based on National Centre for Health Statistics (NCHS) z-scores, among rural adolescents of Paschim Medinipur District, West Bengal.

\section{Materials and Methods}

\section{Study area and subjects}

Data for the present study were collected from higher secondary schools, situated in a rural area within the Paschim Medinipur Sadar north subdivision about $29 \mathrm{Km}$ from Midnapore town. Formal approval was obtained from Vidyasagar University and school authorities prior to the study. Subjects were randomly selected from these schools. The sample size consisted of 1094 (boys $=665$; girls $=429$ ) children aged 11-18 years. Verification of age was done from the school records, as well from the answers to specific questions in the pre-tested questionnaire, which was completed by every subject.

\section{Anthropometric measurements and evaluation of nutritional status}

Height and weight measurements were taken on each subject following the standard techniques ${ }^{17}$ using weighing scale and anthropometer rod to the nearest $0.5 \mathrm{Kg}, 0.1 \mathrm{~cm}$, respectively. Technical errors of measurements (TEM) were found to be within reference values $^{18}$. Thus, TEM was not incorporated in statistical analyses.

Two commonly used undernutrition indicators, i.e., underweight and stunting were used to evaluate the nutritional status of the subjects. The $\mathrm{NCHS}^{19}$ age and sex specific <- 2 z-scores were followed to define underweight and stunting. The following scheme was utilized:

Stunting: height-for-age $\mathrm{z}$-score $(\mathrm{HAZ})<-2.0 \mathrm{sd}$

Underweight: weight-for-age z-score (WAZ) <-2.0 sd

We followed the $\mathrm{WHO}^{2}$ classification for assessing severity of malnutrition by percentage prevalence ranges of these two indicators among children. The classification is:

$\begin{array}{lcccc}\text { Indicators } & \begin{array}{c}\text { Low } \\ (\%)\end{array} & \begin{array}{c}\text { Medium } \\ (\%)\end{array} & \begin{array}{c}\text { High } \\ (\%)\end{array} & \text { Very High (\%) } \\ \text { Underweight } & <10 & 10-19 & 20-29 & \geq 30 \\ \text { Stunting } & <20 & 20-29 & 30-39 & \geq 40\end{array}$

\section{Statistical Analyses}

The distributions of height and weight were not significantly skewed therefore not necessitating their normalization. Between sexes differences in means of height and weight were tested by student's t-test. Oneway and two-way ANOVA were employed to test age, sex and interaction on dependent variables. Odds ratio (OR) and 95\% confidence interval $(\mathrm{Cl})$ were calculated using standard formulae. Proportion test was employed to test the sex difference of underweight and stunting.

\section{Results}

The age and sex specific means $\pm S D$ of weight and height are presented in figure 1 (weight) and figure 2 (height). Results revealed that boys were significantly heavier than girls from age 16 onwards; they were also significantly taller from age 14 years. Results of one-way ANOVA showed that weight and height increased significantly with advancement of age in both sexes. Further, two-way ANOVA tests were employed to determine the effect of age, sex and interaction on dependent variables (Table 1). Except for the weightfor-age and height-for-age z-scores, all anthropometric indicators of nutritional status showed significant effect of age and sex. Except for weight-for-age z-scores, all three dependent variables were significantly influenced by age-sex interaction.

The distributions of weight-for-age and height-forage z-scores are presented in figure 3 and figure 4 in comparison to NCHS standards. Figure 5 and figure 6 presents the age and sex specific mean $\pm S D$ weight-forage and height-for-age z-scores among the subjects. The means in all cases had negative values. Overall, the mean weight-for-age z-scores for boys and girls were -1.488 and -1.417 , respectively. The corresponding mean values for height-for-age z-scores were -1.317 and -1.486 , respectively.

The age and sex specific prevalence of underweight and stunting are presented in Table 2. Based on the z-scores, the rates (age and sex combined) of underweight and stunting were $28.3 \%(95 \% \mathrm{Cl}: 25.7$ - 31.1) and $27.8 \%$ (95\% Cl: 25.2 - 30.5), respectively. The prevalence of underweight was significantly higher 
among boys (31.0\%, 95\% $\mathrm{Cl}$ : 27.6-34.6) than the girls (24.2\%, 95\% Cl: 20.4-28.5). These rates for stunting were $27.4 \%$ (95\% Cl: $24.1-30.9)$ and $28.4 \%$, (95\% Cl: 24.4 - 32.9) for the boys and girls. The rate of underweight and stunting was more among late adolescents (15-18 years) than early adolescents (11-14 years). In boys, the prevalence of stunting was 1.5 times $(\mathrm{OR}=1.47$;
95\% Cl: $1.03-2.09)$ more in late adolescents than early adolescents. According to the WHO classification for assessing severity of malnutrition, the rates (age combined) of stunting were, medium in both sexes. The rates of underweight were high and very high for girls and boys, respectively.



Fig 1: Mean \pm SD of weight $(\mathrm{kg})$ by age and sex.

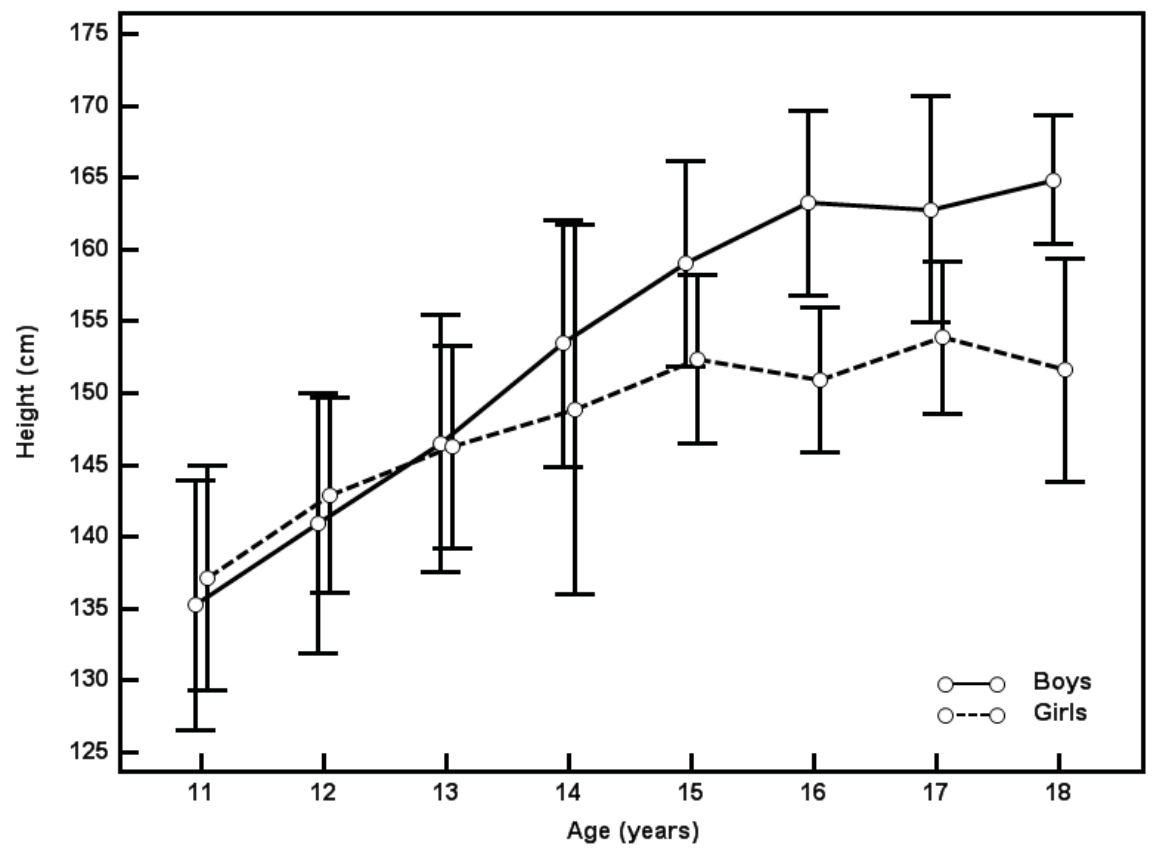

Fig 2: Mean $\pm S D$ of height $(\mathrm{cm})$ by age and sex. 
Table 1: Result of univariate two-way analyses of variances (2-way ANOVA)

\begin{tabular}{|l|c|c|c|}
\hline Dependent Variable & Age (F value) & Sex (F value) & Interaction (F value) \\
\hline Weight & $131.294^{* *}$ & $24.250^{* *}$ & $7.622^{* *}$ \\
\hline Height & $124.631^{* *}$ & $91.898^{* *}$ & $15.603^{* *}$ \\
\hline HAZ & $6.542^{* *}$ & 2.857 & $2.352^{*}$ \\
\hline WAZ & $5.264^{* *}$ & 1.789 & 0.340 \\
\hline
\end{tabular}

${ }^{*} p<0.01,{ }^{* *} p<0.001$. (Age and sex were independent factors)

Table 2: Prevalence (\%) of underweight and stunting by age and sex.

\begin{tabular}{|c|c|c|c|c|c|c|}
\hline \multirow{2}{*}{$\begin{array}{l}\text { Age } \\
\text { (Years) }\end{array}$} & \multicolumn{2}{|c|}{ Sample (n) } & \multicolumn{2}{|c|}{ Underweight (WAZ < - 2) } & \multicolumn{2}{|c|}{ Stunting (HAZ < - 2) } \\
\hline & Boys & Girls & Boys & Girls & Boys & Girls \\
\hline Overall & 665 & 429 & $206(31.0)^{*}$ & $104(24.2)$ & $182(27.4)$ & $122(28.4)$ \\
\hline \multicolumn{7}{|c|}{ Early adolescents: } \\
\hline 11 & 96 & 66 & $26(27.1)$ & $20(30.3)$ & $27(28.1)$ & $15(22.7)$ \\
\hline 12 & 117 & 59 & $33(28.2)$ & $15(25.4)$ & $28(23.9)$ & $14(23.7)$ \\
\hline 13 & 121 & 82 & $36(29.8)$ & $17(20.7)$ & $33(27.3)$ & $27(32.9)$ \\
\hline 14 & 100 & 84 & $31(31.0)$ & $15(19.9)$ & $19(19.0)$ & $23(27.4)$ \\
\hline Total & 434 & 291 & $126(29.0)$ & $67(23.0)$ & $107(24.7)$ & $79(27.1)$ \\
\hline \multicolumn{7}{|c|}{ Late adolescents: } \\
\hline 15 & 70 & 40 & $18(25.7)$ & $8(20.0)$ & $13(18.6)$ & $9(22.5)$ \\
\hline 16 & 56 & 43 & $16(28.6)$ & $11(25.6)$ & $15(26.8)$ & $14(32.6)$ \\
\hline 17 & 47 & 24 & $21(44.7)$ & $7(29.2)$ & $22(46.8)$ & $5(20.8)$ \\
\hline 18 & 58 & 31 & $25(43.1)$ & $11(35.5)$ & $25(43.1)$ & $15(48.4)$ \\
\hline Total & 231 & 138 & $80(34.6)$ & $37(26.8)$ & $75(32.5)^{\star *}$ & 43 (31.2) \\
\hline
\end{tabular}

* Significant sex difference; $p<0.05$.

** Significant age group (late vs early adolescent) difference: $p<0.05$.

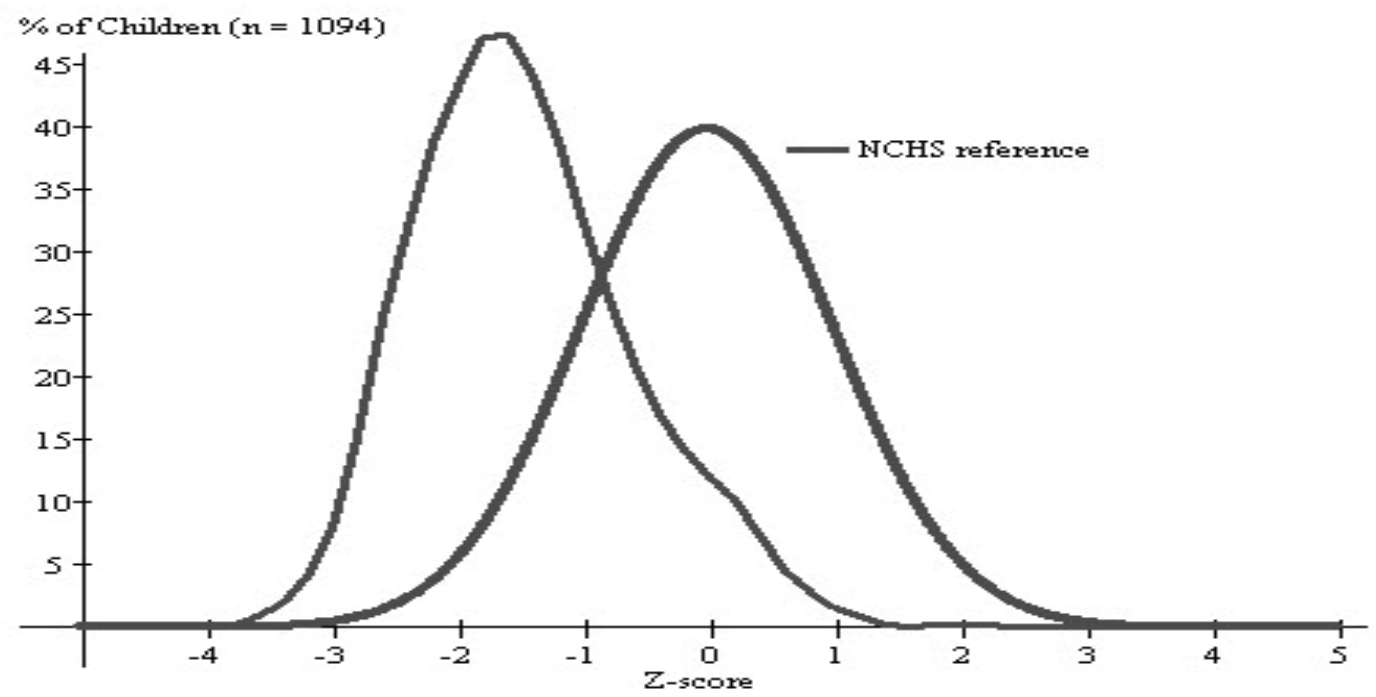

Fig 3: Distribution of weight-for-age z-scores of the rural adolescents 


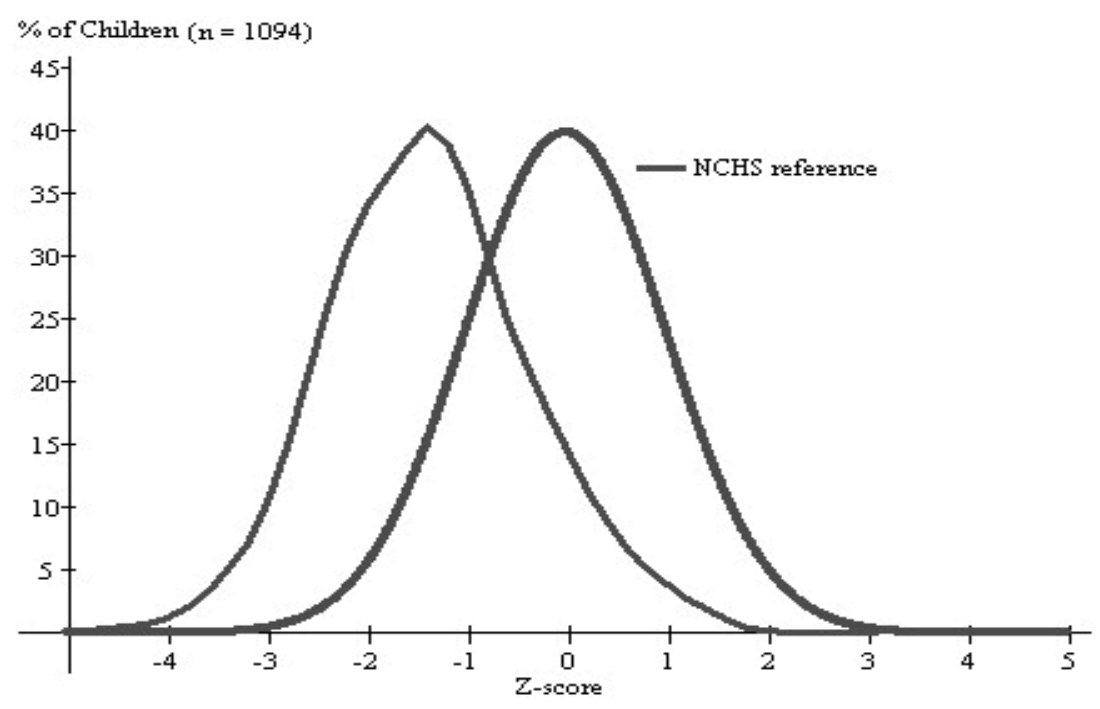

Fig 4: Distribution of height-for-age z-scores of the rural adolescents

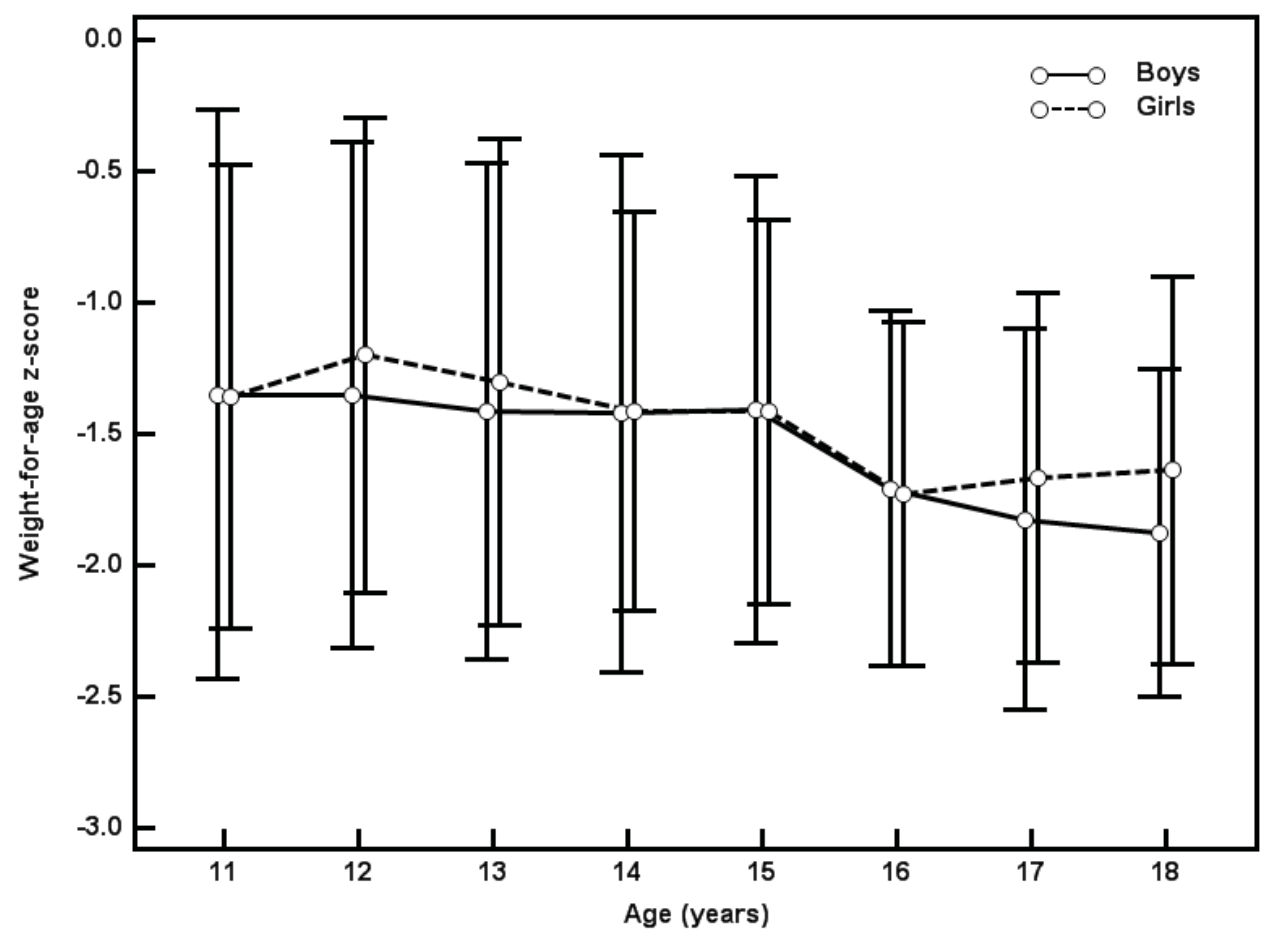

Fig 5: Mean $\pm S D$ weight-for-age z-scores by age and sex

\section{Discussion}

Anthropometric indicators have been widely used in population-based studies directed to nutritional evaluation. Although they are proxy indicators, that is, they represent indirect measures of undernutrition that do not take into account nutrient intake or biochemical examination, their wide use is justified due to the ease of the method and its high sensitivity to nutritional alterations in a population ${ }^{20}$. Cohort studies, ideal for nutritional conditioning monitoring, suffer, in developing countries like India, from the logistic difficulties usually associated with population studies of large magnitude. In such cases, cross-sectional studies can provide relevant information for understanding the connection between health status and physical conditions of life. These studies have the advantage of relatively low costs and they can also provide fundamental information for 




Fig 6: Mean \pm SD height-for-age z-scores by age and sex.

the implementation of health surveillance systems and the definition of long-term health intervention strategies $^{21,22,23}$.

Undernutrition continues to be a cause of illhealth and premature mortality among children and adolescents in developing countries like India ${ }^{24,25}$. The two most commonly used indicators of undernutrition among adolescents are underweight (low weight for age) and stunting (low height for age). Stunting is an indicator of chronic undernutrition, the result of prolonged food deprivation and/or disease or illness whereas underweight is used as a composite indicator to reflect both acute and chronic undernutrition, although it cannot distinguish between them².

These indices are compared against an international reference population developed from anthropometric data collected in the US by the $\mathrm{NCHS}^{19}$. Children whose measurements fall below -2 z-scores of the reference population median are considered undernourished, i.e. to be underweight or stunted. These indices reflect distinct biological processes, and their use is necessary for determining appropriate interventions ${ }^{2}$.

The results of the present study clearly indicated that, based on WHO classification of severity malnutrition, the (age combined) prevalence of underweight was high (20-29\%), while those of stunting were medium (20 $29 \%$ ), in both sexes. The rates of underweight, which is used as a composite indicator to reflect both acute and chronic undernutrition, demonstrated that the level was high. In case of stunting among these subjects, there existed medium level of chronic undernutrition due to prolonged food deprivation. Overall, these findings suggested widespread adverse nutritional experience of the subjects.

It has been suggested that since undernutrition is a function of both food deprivation and disease, which are in turn the consequences of poverty, anthropometric indices can serve only as proxies for evaluating the prevalence of undernutrition among children ${ }^{25}$. Efforts to reduce undernutrition, morbidity and mortality depend on reducing poverty and raising people's living standards by improving the quality of homes and by increasing access to clean drinking water and adequate sanitation. Such interventions have positive impacts on health, and implementing these also goes some way towards fulfilling people's basic human rights ${ }^{25}$. Since the nutritional status of the subjects of the present study is not satisfactory, it seems that there is scope for much improvement in the factors associated with their nutritional status. The school health services might provide an ideal platform to detect the health problems early and treat them. Early detection of the morbidities through regular survey helps in prompt treatment and prevention of serious health complications ${ }^{7,26}$. Therefore, such kind of studies may help to formulation of appropriate strategies to combat health complication of adolescents that already an important global public health burden in the last two decades. 


\section{Acknowledgements}

The school authorities of Khasla Indumati High School, Salboni, are gratefully acknowledged for their help and cooperation. We would also like to thank all the subjects who participated in this study.

\section{Funding}

This work was done with the financial assistance of University Grants Commission (Government of India) Major Research Project Grant Number F. 5-127/2004 (HRP).

Conflict of Interest: None

Permission from IRB: Not Required

\section{References}

1 World Health Organization. Adolescent Nutrition: A review of the situation in selected South-East Asian countries. Executive Summary. World Health Organization Regional Office for South-East Asia, New Delhi, March, 2006.

2 WHO. Physical status: The use and interpretation of anthropometry. Technical report series. Geneva; World Health Organization; 1995. Report No.: 854.

3 Bisai S. The relationship of maternal anthropometry and birth outcome among Bengalis of Kolkata. IDSK Working Paper Series - 4. April, 2009.

4 Kirchengast S, Winkler EM. Nutritional status as indicator for reproductive success in Kung San and Kavango females from Namibia. Anthropol Anz 1996;54(3):267-276.

5 Thame M, Wilks RJ, McFarlane-Anderson N, Bennett FI, Forrester TE. Relationship between maternal nutritional status and infant's weight and body proportions at birth. Eur J Clin Nutr 1997; 51(3): 134-138.

6 Goyal RC, Chavan UA. Health status of school children in Ahmednagar city. Indian J Matern Child Health. 1993;4(3):81-83.

7 Dambhare DG, Bharambe MS, Mehendale AM, Garg BS. Nutritional Status and Morbidity among School going Adolescents in Wardha, a Peri-Urban area. OJHAS 2010;9:(2).

8. Lee RD, Nieman DC. Nutritional Assessment. New York: McGraw Hill, 2003.

9. Venkaiah K, Damayanti K, Nayak MU, Vijayaraghavanm K. Diet and nutritional status of rural adolescents in India. Eur $J$ Clin Nutr 2002;56:1119-1125.

10. Khongsdier R, Varte R, Mukherjee N. Excess male chronic energy deficiency among adolescents: a cross-sectional study in the context of patrilineal and matrilineal societies in Northeast India. Eur $J$ Clin Nutr 2005;59:1007-1014.

11. Deshmukh PR, Gupta SS, Bharambe MS, Dongre AR, Maliya C, Kaur S, Garg BS. Nutritional status of adolescents in rural Wardha. Indian $J$ Pediatr 2006;73:139-141.

12. Rao, KM., Laxmaiah, A., Venkaiah, K. and Brahman, GN. Diet and nutritional status of adolescent tribal population in nine states of India. Asia Pac J Clin Nutr 2006; 15: 64-71.

13. Bose K, Mukhopadhyay, A. Nutritional Status of Adolescent Bengalee Boys. Indian Pediatr 2004;41:633,2004.

14. Das DK, Biswas R. Nutritional status of adolescent girls in a rural area of North 24 Parganas district, West Bengal. Indian J Pub Health, 2005;49:18-21.

15. Mukhopadhyay A, Bhadra M, Bose K. Anthropometric Assessment of Nutritional Status of Adolescents of Kolkata, West Bengal. J Human Ecol 2005; 18: 213-216.

16. Bose $\mathrm{K}$ and Bisai S. Prevalence of underweight and stunting among school children aged 6-18 years in Paschim Medinipur, Bankura and Puruliya districts of West Bengal. Indian J Pediatr 2008;75 (12):1272.

17. Lohman TG, Roche AF, Martorell R. Anthropometric Standardization Reference Manual. Chicago: Human Kinetics Books, 1988.

18. UlijaszekSJ, KerrDA.Anthropometric measurement error and the assessment of nutritional status. $\mathrm{Br} \mathrm{J}$ Nutr 1999; 82: 165-177.

19. World Health Organization. Measuring Change in Nutritional Status. Geneva: World Health Organization, 1983.

20. Post CL, Victoria CG. The low prevalence of weightfor-height deficits in Brazilian children is related to body proportions. J Nutr 2001;131:1133-1134.

21. Waterlow JC, Buzina R, Keller W, Lane JM, Nichman MZ, Tanner JM. The prevention and use of height and weight data for comparing the nutritional status of group of children under the age of 10 year. Bull WHO 1977; 55: 489-498. 
22. Satyanarayana K, Radhaiah G, Mohan KR, Thimmayamma BV, Rao NP, Rao BS, Akella S. The adolescent growth spurt of height among rural Indian boys in relation to hildhood nutrition background: a 18 years longitudinal study. Ann Hum Biol 1989;16:289-300.

23. Martorel R, Rivera J, Kaplowitz H. Consequences of stunting in early childhood for adult body size in Guatemala. Ann Nestle 1996;48:85-92.

24. Gopalan, C. Nutrition Research In South-East Asis: The Emerging Agenda of the Future. Regional
Publication, SEARO. No. 23. Geneva: World Health Organization, 1996.

25. Nandy S, Irving M, Gordon D, Subramanian SV, Davey Smith G. Poverty, child undernutrition and morbidity: new evidence from India. Bull WHO 2005;83:210-216.

26. Panda P, Benjamin AI, Singh S, Zachariah P. Health status of school children in Ludhiana city. Indian J Comm Med 2000;25(4):150-155.

\section{How to cite this article?}

Bisai S, Bose K, Ghosh D, De K Growth Pattern and Prevalence of Underweight and Stunting Among Rural Adolescents. J Nepal Paedtr Soc 2011;31(1):17-24. 